which is most clearly made out. I know of no disease the series of causes and effects in which is so perfectly displayed to the philosophical physician.

In the third place, epilepsy has been more than any other disease delivered over to empiricism, both in and out of the profession. Heberden observes-"Innumera remedia quae in libris medicis celebrantur, usu multo fretus, magnopere laudare non possum."

I was called two days ago to see a poor boy, thirteen years of age, who had been subject during the whole of his life to epilepsy; it had begnn in teething, and had been continued from the various emotions, mental and bodily efforts, and the various irritations, to the present date. Ho had been treated by physicians and by empirics, by mesmerism and by homco. pathy, by silver and by zinc, \&c. \&c. In another patient, the chief remedy had been Baron Sloet's powder; in a third, the cotyledon unibilicus.

It is not my intention to write a criticism upon this matter; but I think I may be permitted to say that those who have attended to the recent progress made in our knowledge of epilepsy will be least inclined to trust the welfare of their patients to such inadequate, incommensurate empiricism. They will rather ask what is the cause, or what are the causes, of the given case of epilepsy, and avoid them; what are the effects already induced by it, and remedy them; in what does the susceptibility to its attacks consist, and eradicate it. Such, at least, will be their rational aim.

In the fourth place, epilepsy is only one of a Class of cognate diseases;-infantile convulsion, puerperal convulsion, apoplexy and paralysis, and mania, inorganic in origin, and paroxysmal in form; \&c. \&c. Who does not feel the necessity incumbent upon him of investigating and treating such cases rationally? Who would rest satisfied with a blind empiricism in such cases? Then why should we yield up our faculties in the cognate case of epilepsy?

In the fifth place, epilepsy assumes forms which are at once the mildest and the most transitory, and the direst and most lasting of maladies. It may consist in a brief oblivium; or it may issue in a fit of mania, in paralysis or spasmo-paralysis, or in dementia. It is something to be able to convert the graver into the milder malady, the epilepsia laryngea or gravior into the epilepsia trachelea or mitior, or abortiva, could we do nothing more. This may be accomplished by tracheotory?

But more may follow. The severer form of the disease being prevented, not only may its more formidable effects be prevented, but the excitability of the spinal centre and the susceptibility to future attacks may wane away and cease!

In one case, rejected by the wisdom and benevolence of the Royal Medical and Chirurgical Society, the most formidable attacks of epilepsy, with danger to life, have been totally superseded; the patient's life was in the utmost jeopardy, and was saved; the poor patient himself, a boatman, has been rescued from all returns of epilepsy, and enabled to pursue his avocation without the danger of falling into the water, and being drowned! Was ever case more worthy of record ? In another case epilepsy laryngea has been prevented; threatenings occur, but are abortive; these threatenings are becoming more and more slight, and the patient's health and intellect are both improved! In a third case, in which the patient, aged thirty-six, has been afflicted with hereditary epilepsy during twenty-four years, of the direst kind, scarcely constituting a case for the trial of any remedy, the fits have assumed the milder form.

Is there not enough in all this to excite a hope, an enthusiastic hope, that much still remains to be accomplished and explored, in reference to this important measure in this formidable disease? Are there on medical record cases of deeper interest?

Here I terminate these remarks for the present, and do so begging my readers to consider how much has really already been achieved in this investigation. The fact of the movement of the separated tail of the triton on being irritated, cursorily observed, has led to the detection of the physiology of all the functions of ingestion and of egestion in the animal economy, and of the pathology of the entire Class of spasmodic diseases!

ST. LuKE's Hospital. - We have great pleasure in announcing that arrangements bave now been made for students to witness on certain days the practice of this hospital. It may not perhaps be generally known, that the medical board of the East India Company now require an attendance of three months at a lunatic hospital, from candidates entering their service in a medical capacity.

\section{A DESCRIPTION OF \\ TWO CASES OF EXTROVERSION OF THE BLADDER;}

WITH AN EXPLANATION OF THF ERROR OF DEVELOPMENT FROM WHICH SUCH MALFORMATIONS MOST PROBABLY ARISE.

Br EDWARD I. CHANCE, Ese, F.R.C.S., SENIOR SURGEON TO THE METROPOLITAN FRER HOSPITAL, SURGEON TO THE CITY ORTHOPADIC HOSPITAL, ETC.

Althougin cases of extroversion of the bladder are far too severe malformations to permit us to entertain the hope of effecting a cure by surgical treatment, they nevertheless present sufficient points of interest to the surgeon and physiologist to warrant my obtruding before your numerous readers the particulars of two instances of the kind which are under my care at the present time. To the surgeon, such cases are interesting, because, hitherto, as far as I am aware, they have entirely baffled every attempt at treatment. To the physiologist, however, they are still more so, because the agreement between them is so great, that it proves such malformations are not the mere result of accidental erroneons development, but arise from some specific error, acting invariably in the same manner, the nature of which error has not as yet been exactly determined.

From the preceding observations, it will be expected that the cases $I$ am about to narrate should possess this absolute similitude of character; an expectation which will be fully borne out, inasmuch as, with two minor circumstances, the description of the one would do equally for that of the other.

The first case is that of an infant, at present only a few weeks old. It was sent to me by Dr. T. L. Blundell, twelve hours after its birth, at which time it presented the same appearance as at the present moment, excepting that the funis was then attached to the upper part of the protrusion.

The second case is that of a lad, thirteen years of age, who presented himself to me as a patient at the City Orthopædic Hospital, Hatton garden.

Upon examining these cases, the first point which attracts attention is the red, fleshy mass protruding from the abdomen at the normal situation of the upper edge of the symphysis pubis. This protruding mass appears to arise from a kind of cicatrix which surrounds it at the sides and above; while below it rests upon a full-sized but malformed penis, which immediately surmounts the scrotum, and therefore occupies its normal position. In each case the testicles have descended, and are contained within the scrotum.

Upon further and more careful examination, it will be found, that upon raising the protruding mass from off the penis, the latter organ is divided along its entire length from above, down to the urethra, (epispadias,) so that the urethra resembles a groove or gutter on its upper surface, the glans penis being spread out at its extremity on either side, beneath which a thick fold of skin corresponds with the divided prepuce; indeed, it has just the appearance of this organ after the operation for phymosis. On tracing the urethral groove backwards, we find it connected directly with the fleshy mass by a fold of membrane, half an inch in length, occupying the situation of the caput gallinaginis; and on either side of this membranous fold, at the spot where it joins the tumour, we distinctly recognise the existence of a band of muscular fibres, passing off laterally for about half an inch, and diverging from each other on the tumour, where they are joined by a still more distinct cross-band, thus converting the space bounded by them into a triangle, (the "trigone vesicale,") from the upper angles of which the urine is seen to be almost constantly exuding in a minute, dribbling stream, or occasionally, in the infant, upon an effort at straining being made, in a very fine but continuous jet, that is projected some distance from the body.

From the preceding description, it will be at once recognised that the protruding red mass is no other than the bladder, which is abnormally deficient of its anterior parietes, turned inside out, or extroverted by the pressure of the viscera behind it, and protruded out of the abdomen in the resemblance of a fungons growth, in consequence of the absence of the whole of the parts which are normally situated anteriorly to it. In both cases, the appearance of the extroverted bladder is alike, it being highly vascular, covered with a thick, tenacious, and transparent mucus, and its surface either slightly corrugated or quite smooth, according as it is in a state of repose, or pressed upon and therefore further pro. truded by the action of the abdominal muscles and diaphragm on any extra exertion of those parts, such as crying, \& c. The 
only visible point of difference in the appearance of the bladder in these two cases is, that in the one (the infant) the ureters (of which there are three, two on one side, and one on the other) terminate in distinct papillæ, which project the one-sixteenth of an inch, and rest one on either side against the glans penis, ${ }^{*}$ and the third half-way up on the right side; whereas, in the elder case, the spot at which they terminate cannot be detected, except at the moment the urine is flowing, when it becomes visible at the bottom of a minute pit or depression, caused by the traction upon the extremities of the ureters by the muscular fibres leading from them to the neck of the bladder, which depression, moreover, is immediately afterwards elevated to the level of the surrounding surface.

As the anterior part of the bladder is deficient, and the urethra open throughout its whole course, it is most interesting to observe the change in the form and situation of the various parts, from that which they possess in the normal condition of the organs. The situation of the orifices of the ureters, and the existence of the trigone vesicale, as well as the fold of membrane occupying the place of the caput gallinaginis, have been already described, but I must not pass by these parts without recording the fact, that although the imperfect neck of the bladder, in these unfortunate cases, has not to perform its natural function of regulating the discharge of the urine, nevertheless the spot itself retains all the exquisite sensibility natural to it in its perfect state; and it is still, although it has no function to perform, by far the most sensitive part of the whole exposed surface.

The membranous portion of the urethra and bulb are altogether absent, or if there be any portion of these parts developed, they are so short that their limited extent cannot be defined; the urethra therefore consists of the prostatic portion and of that part which traverses the body of the penis, altogether about an inch and a guarter to two inches in extent, the former being the length of the urethra of the infant, the latter of the older patient.

In each case, upon the surface of the urethra you can readily detect the lacuna magna, the sinus pocularis, and the openings of the ejaculatory ducts. The prostate appears very rudimentary, and is of course deficient of the portion ordinarily situated above the urethra.

In describing the situation of the extroverted bladder, I have stated that it occupied the normal position of the upper edge of the symphysis pubis; but in the cases at present under consideration no such articulation exists, as the pelvis in each case is imperfect anteriorly, the pubic bones being widely separated from each other, and in all probability malformed.

In the elder case the crest of the pubes can be plainly discerned by the finger, at the spot indicated on the wood-cut by the mark 3, and the interspace between them can be as readily and plainly distinguished; in the infant the angles of the pubic bones are not so distinctly to be felt; but this is not to be wondered at, seeing that at such an early age ossification of these parts has not commenced. The interspace between them is, however, equally plainly to be discerned; and upon one occasion I passed the extroverted bladder back into the abdomen upon the point of my finger.

The examination of the external configuration of the abdomen affords to the eye of the anatomist additional evidence of the abnormal position of the pubic bones, as the recti muscles, instead of running throughout their whole course parallel to one another, merely separated by the linea alba, are plainly to be observed, about midway, to begin to diverge from each other, and to get farther and farther apart, until they reach their insertion in the crest of the pubes, (see wood-cut, 3, leaving between them a distinct triangular depression instead of the strictly linear " linea alba."

The abnormal triangular interspace just noticed, is bounded on each side by the internal edges of the recti muscles, and extends superiorly from a point which corresponds exactly with the normal situation of the umbilicus, (see Fig. 1-1,2,) down to the extroverted bladder, which occupies and forms the base of the triangle, on a line which corresponds with the situation of the symphysis pubis in the perfect skeleton.

As the pubic bones are thus widely separated from each other, and in all probability merelv united by ligamentous union, the pelvis, as a whole, necessarily lacks that unyielding firmness which is requisite to give stability to the movements of the body in progression; and we accordingly find, in the elder sufferer, that in walking, each step is taken with evident care, and with a slightly bent or stooping attitude.

From the account just given of the condition of the pelvis

* A very much larger quantity of urine flows from the orifice of the left ureter than from both of those on the right side together.

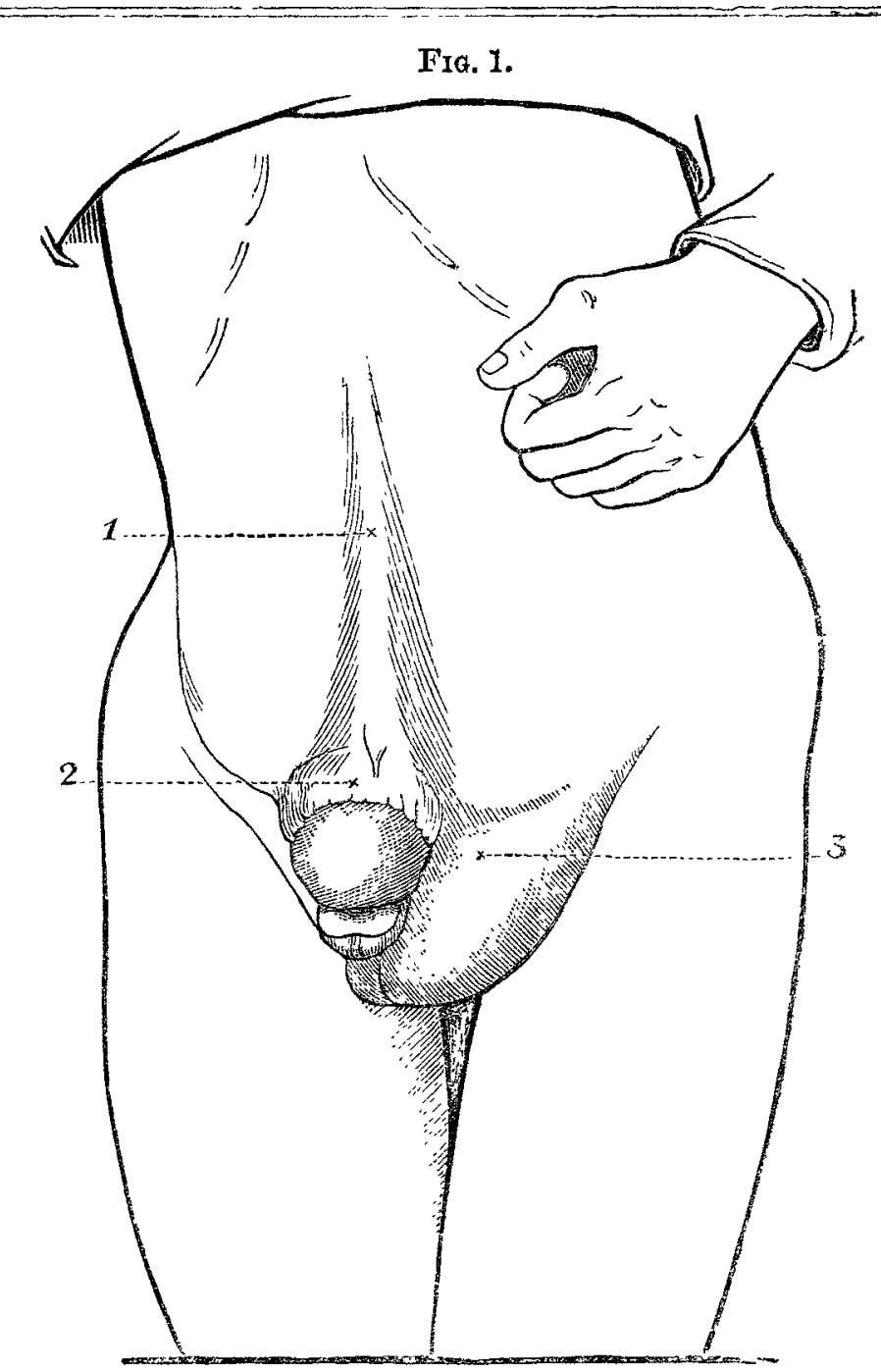

Fra. 2.

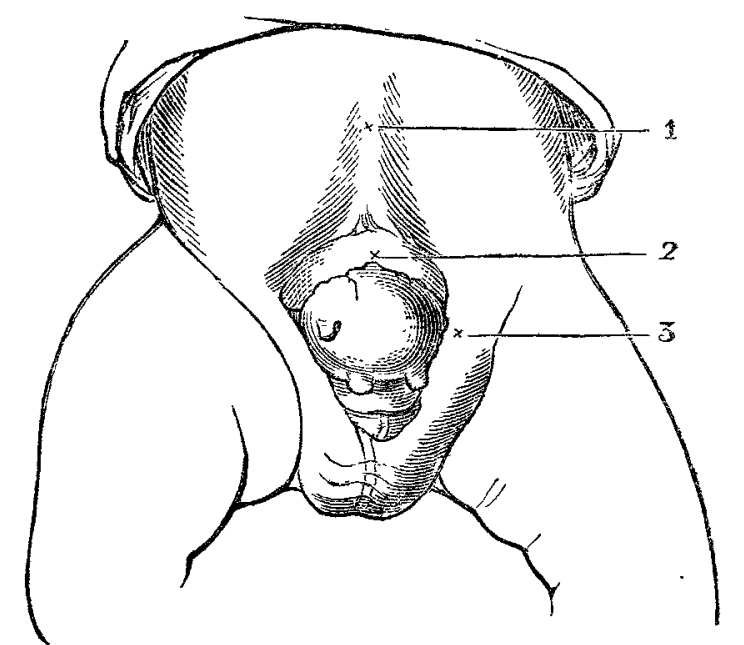

Fig. 1.-Drawing of the elder patient.

(The references are the same in both.)

1 , the normal situation of the umbilicus; 2 , the spot from whence the umbilical vessels were separated, or abnormal umbilicus ; 3 , the crest of the pubes.

and abdominal muscles, and consequent relaxation of the parts about the groins, it will be hardly deemed a matter of surprise that the elder lad should also be afflicted with con. genital hernia on both sides; at the time the drawing for the wood-cut was made, the hernia was much larger on one side than on the other, but occasionally the right is as large as that on the left. In the younger case, although there is great fulness in each groin, there is no hernia.

There is yet one other point of deep interest that $I$ have purposely omitted to notice until the last, for the reason that it appears to me to afford the direct key to the comprehension of the mode in which these sad malformations are developed I allude to the situation of the umbilicus, which in both cases, (as also in two others which $I$ have seen in the course of 
my practice,) instead of occupying its normal position, is observed to be situated at the upper part of, and conjoined with, the extroverted bladder just above the pubes, (see Fig. 2-1, 2, ) thereby constituting a part of the kind of cicatrix to which I alluded.

In the infant $I$ had the opportunity of observing these parts while the umbilical cord was yet attached, consequently no doubt or ambiguity exists as to the precise spot at which it made its exit from the abdomen; and in this case, now that it is separated, and the parts healed, the appearance is, as near as can be, in every respect like the same parts in the elder patient.

The spot at which the umbilical cord normally proceeds from the abdomen is about midway between the ensiform cartilage and the symphysis pubis-or, in other words, from the precise spot we should theoretically expect the vessels composing it to pass out if we take into consideration the fact that they are either derived from, or are proceeding to, vessels situated at the upper and the lower part of the abdominal cavity, and therefore that in the closure of the parietes of the abdomen during the development of the embryo, they are surrounded and covered in by them gradually, but equally above as well as below, their point of emergence being the apex of a triangle, the base of which is constituted by the spine and large vessels to which they are connected.

If this expectation be correct, it necessarily follows that such an alteration in the situation of the umbilicus, as that which I have described, could not occur, except from the action of causes which prevent the development of the abdominal parietes below, while it is permitted to progress uninterruptedly above; under which circumstances the umbilicus wonld of necessity be proportionally removed from its usual position. As the vessels of which the cord is chiefly made up belonged originally to the allantois, from which latter organ is also developed the urinary bladder, it appears to me that we have here the direct explanation of the mode in which these malformations are built $\mathrm{up}$, although we may not be enabled to discover the direct or proximate cause itself.

In the development of the embryo, according to Bischoff, the allantois is discernible before there is any trace of either the intestinal tube or of the Wolffian bodies; and, according to Wagner, when the embryo is only two lines in length it is already developed into a distinct vesicle leading from the caudal extremity of the embryo. See woodcut 3 , copied from Wagner.

Fia. 3.

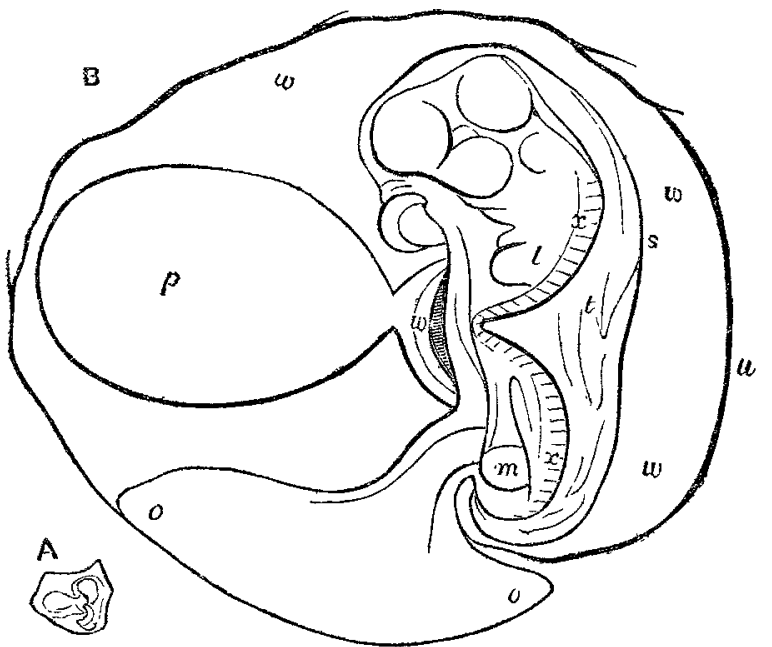

Fia. 3.-A, human ovam of the third week, laid open, and exposing the embryo-natural size; $B$, the same ovam magnified $u$, chorion laid open, and reflected; $w w w$, albuminous space betwixt the amnion and chorion; $s t$, the amnion; $l$, the anterior, and $m$, the posterior extremities; 00 , presumed limits of the lamina vasculosa of the allantois; $p$, umbilical resicle; $x x$, the vertebral column.

The office of the vesicular allantois is twofold; in the first place it conducts the bloodvessels of the embryo to the chorion to form the placenta, and therefore the trunks of the vessels so conducted afterwards become the identical vessels which constitute the umbilical cord; and, in the second place, it is directly modified so as to form, by its dilatation, the urinary bladder, from the upper part of which there proceeds the still tubular portion known as the urachus, while from the lower extremity two processes pass backwards, which become the ducts of the Wolffian bodies, (afterwards the ureters;) and as growth progresses, a yet more inferior process is developed, which becomes converted into the urethral canal. The umbilical cord and the bladder are therefore, at this stage of development, in close apposition.

As these are the undoubted offices performed by the allantois, it appears to me that malformations of the bladder, \&c., such as $I$ have described, are the direct result of some error, such as over-distention of the allantois by air or fluid, which is capable of giving rise, in the early stages of development, to a minute aperture or rent on its anterior surface, which, by simple enlargement in the longitudinal course of the yet delicate vesicular tube, lays it entirely open throughout its whole course-i. e., upwards through the urachus to the vessels of the cord, and downwards through the urethra to the extremity of the penis; hence entirely preventing the development of the anterior portion of the bladder, of the prostate, and of the urethral canal.

At this early period, although the vertebral column is distinctly marked out, and the extremities clearly discernible as "leaf-like elevations," the arch of bones which is requisite to support them is not as yet completed anteriorly; in which circumstance we have the direct explanation of another portion of the malformation - viz., the permanent incompleteness of the pelvis; for, as the urethra normally passes beneath the arch of the pubes, if a rent of the allantois exists at that part which corresponds to, and after. wards constitutes, the urethra and prostate, it is impossible for the pubic arch ever to be developed across $i t$, as there wonld necessarily result from that rent, a want of continuity in the membrane which conducts the blastema, or in the blastema itself; consequently the basin of the pelvis must remain, as in the present cases, imperfect at its anterior portion, and the union of the pubic bones be effected in some irregular mauner beneath the now exposed urethra, instead of meeting above it in the mesial line as the symphysis. I am aware that in some few of the cases that have been described, the pubic symphysis is stated to have been complete; but as these instances were, to the best of my belief, adults, it is just possible that in these exceptions to the generally recorded experience, ${ }^{*}$ if they had been examined at an early period of life, the absence of the symphysis would have been discernible; and that at or about puberty a spurious spmphysis had been formed beneath and behind the urethra and bladder by the process of ossification spreading along the ligamentous structure by which the bones were united.

If the rent in the allantois does not include the portion which is subsequently developed into the urethra, in that case the development of the pelvic arch would not be inter fered with, and the urethra would then occupy its normal position, and the penis be free from deformity.

There is another circumstance, however, which confirms me in this opinion-viz., that as the bladder in these cases protrudes between the recti muscles, if the pubic symphysis with its attached muscles existed, the bladder could not fail to be compressed laterally every time the recti muscles were put into action, whereas no such compression is experienced, or has been noticed; but, on the contrary, in the cases detailed in this paper, there is an absolute and well-marked triangular interspace, demonstrating the separation of their inferior insertions from each other.

The alteration in the position of the umbilicus, the consideration of which has led us to the explanation of these malformations, is therefore just what we might anticipate from the effect of a rent in the allantois; as the integument of the abdomen and the linea alba above the cord would proceed regularly and uninterruptedly in their development; whereas below the cord the development of these parts would be effectually prevented, as the portion of the allantois forming the urinary bladder could not be withdrawn into the abdomen at its proper period, in consequence of the fissure preventing the development of the pubic arch in front of it; consequently the umbilical vessels remain, as when first developed, in absolute contact with the upper part of the urinary bladder; and the umbilicus-i. e., their point of exit-becomes permanently fixed in the hypogastric region by the closing in of the integument and linea alba above and around them. That the umbilicus must occupy the hypogastric region instead of its normal position in all cases in which the bladder is extroverted, is easily demonstrated by diagrams alone; but $I$ will not at this time further intrude upon your valuable space.

The imperfection in the urethra and penis noticed in these cases is known under the term Epispadias, in contradistinction to another and opposite lkind of irregularity of development, in which the urethra is open for a greater or less extent onits under surface, in which case it is called Hypospadias.

* In by far the greater number of cases the bones are said to have been eparated. 
As I have attempted an explanation of the former malformation, I will here, for the sake of endeavouring to render the subject complete, briefly allude to the cause of this latter imperfection, especially as it proceeds from an error of an entirely different nature.

In the growth of the embryo, the various parts of the body progress through different phases of development, from the mere rudimental cell to the perfect organ; and it is wellknown that occasionally an arrest of development in a particular organ occurs while every other part of the body progresses regularly on to perfection.

The result of this error is, that the merely rudimental and ordinarily transient form of an organ becomes affixed to it for ever; and when such is the case, the deformity may be accurately described as being the result of an " arrest of development."

In the development of the organs of generation, the penis, up to the third month, has the urethra open all along its under surface; or rather it has a groove, the margins of which, about the fourteenth week, begin to close up and form the urethra.

As such is the acknowledged condition of the urethra, by the best authorities, up to the third month, if by any accident the union of the margins of this groove is wholly or in part prevented, the deformity called hypospadias is the direct result. In this instance, therefore, it is perfectly correct to say that the deformity results from "an arrest of development;" but this expression is altogether erroneous when it is applied to such cases as epispadias, or extroversion of the bladder, because at no period of embryonic life is the urethra open above, or the bladder deficient of its anterior moiety.

Old Broad-street, City, 1852.

\section{A CASE OF HOUR-GLASS CONTRACTION OF THE UTERUS.}

By W. H. BOREAM, Esq., M.R.C.S., \&c.

Midwifery is a branch of medical science that should, above all othere, lay claim to our best attention and strictest research, as it involves the life or death, happiness or misery, joy or sorrow, of families; and it is with feelings of the fullest approbation and pleasure that the community will appreciate the excellent arrangements of the Council of the College of Surgeons, by instituting an examination in this important branch, (at the same time the public will bewail the recent loss of one of the brightest ornaments and authorities that ever practised obstetrics.*) Perhaps the following rare case may not be deemed inappropriate:-

Mrs. Mary-Ann G-, aged twenty-three years, a finegrown, well-looking woman, a little above the ordinary height, freckled, and having a profusion of dark-brown hair, was taken in labour on the 22nd of November. She had experienced more or less pain since six o'clock A.M. At six P.M., $I$ was requested to attend her. Her abdomen was unusually large and pendulous. On examination per vaginam the os uteri was just within the reach of the finger, situated opposite about the third transverse line, in the anterior surface of the sacrum; it looked obliquely backwards and downwards, thus showing a little anteversion of the uterus; it was bnt slightly dilated about the circumference of a sixpence. She was left until half-past nine P.M., when I called again, and found the dilatation extended to about the size of a child's india-rubber ring, and as elastic; the pains had rectified its position. The amniotic wedge was gradually protruding, and preparing, by gentle extension, the whole circle of the os tincre to meet its full dilatation. In less than twenty minutes the membranes burst, and an unusual quantity of liquor amnii gushed out, and the expulsion of the child was rapidly accomplished, by two very powesful pains, at five minutes past ten o'clock P.M. The patient now complained, and said that her secondary pains were different in character from those of her former labours; they were gnawing, and griping; "coming in one hip-bone and going out of the other" was her language. There was little or no intermission or expulsive power in them; the pulse was quick 105 , and feeble; the face was anxious in expression and blanched; with cold sweats upon the nose and lips; breathing a little laboured. The abdomen was exceedingly flabby and fat, pursed up into transverse folds; and on examining the uterus through this corrugated wall, and grasping it, it conveyed to the touch the sensation as if squeezing a quart indian-rubber enema syringe filled with fluid. On making a vaginal examination, I found hoemorrhage proceeding to an enormous extent; as much as three pints came during the time I was securing and dividing the umbilical cord. The blood still continuing to flow, and the patient not experiencing any regular uterine contraction, $I$ introduced $\mathrm{my}$ hand into the uterus, and found this unusual constriction. This organ consisted of two chambers: the lower was formed by the cervix and greater inferior half of the body of the uterus, and the upper chamber was formed by the remaining superior portion of its body and fundus, the constriction being about two-thirds from the os; the lower chamber was the deepest, and situated in the true pelvis, the upper appearing very shallow, and located in the iliac or false pelvis. The placenta was grasped by the contraction, and encysted in both chambers, the lower containing the umbilical and larger portion of it. Its substance was very friable in its nature, and easily lacerated. I withdrew its greater proportion, but could not extract the uterine part, for it was morbidly adherent to the fundus, and, for a still better reason, the constriction would only allow but of one finger to pass it, and then firmly contracted around it like a strong sphincter muscle. After the removal of the umbilical portion of the placenta, (and although the remaining portion was still incarcerated in the upper chamber, the hremorrhage gradually abated, and I gave my patient a mixture containing compound spirit of ether, dilated sulphuric acid, syrup of poppies and camphor mixture, and bandaged the abdomen tightly, and ordered the child to the breast; and the uterus now soon became harder and more contracted in its general outline. During the following thirty-six hours, six napkins were thoroughly saturated with blood; the secondary pains were still irregular, and almost without intermission, and I gave her fifteen grains of compound ipecactianha powder, and repeated the mixture; and after about forty hours the remaining portion of the placenta came away. The milk then soon flowed into the breasts, and the patient became comparatively easy, and is now, with her infant, doing well, convalescent.

Remarks.-There seems to have been a great diversity of opinion about the seat of stricture in the uterns in hour-glass contraction; therefore I took great pains in carefully and accurately manipulating it, so that I might form a correct opinion from actual practice; and this must plead an excuse for my troubling you with the case.

The circular fibres in the body of the uterus were generally supposed in such cases to be spasmodically contracted, and my case seems to support that view. But I cannot divest myself of the idea that the slight auteversion of the uterus and pendulous state of the abdomen might have, in some measure, produced this state, as the body of the uterus was most probably, in the latter months of pregnancy, resting on the horizontal rami and symphysis of the pubes, and the uterus thus becoming doubled, or bent uniformly with the body of the child, with its fundus in the abdomen and its neck in the pelvis, and thereby some injury done to the muscular coats of the body of the uterus from pressure, rendering them liable to unequal contraction. The longitudinal fibres in the posterior part of the body of the uterus beivg overstretched, and the anterior ones rendered inert from pressure, and the circular ones unimpaired in strength, gave the maximum of power to the circular ones; and my views of the position of the child receive further support from the fact, that the umbilical cord gave way upon the slightest traction in a place where it was less developed than in any other part of its conrse, seeming as if it had received pressure; and the child was born in that position, where the os frontis and face emerged, looking under the arch of the pubes, and with its back to the anterior surface of the concavity of the sacrum; and the child moreover was meagre, and seemed to have suffered from defective nutrition, and the placenta was unusually large and congested. My idea of the cause of the hour-glass constriction, as above narrated, has never, as far as my reading has extended, been advanced as a reason for it. Dr. E. Rigby, in his "System of Midwifery," page 224, says-" From observation of late years there is much reason to suppose that the true hour-glass contraction, as now described, is of very rare occurrence, even if it does take place at all; and that in by far the great majority of cases the stricture is either produced by the upper part of the cervix, as we have already mentioned, or resides in the os uteri externum, or inferior portion of the cervix." Baudelocque, Dr. Douglas, of Dublin, and the late J. W. Schmitt, of Vienna, he says, were of opinion that its seat was in the neck of the uterus; "and," continues the writer, "from our own experience we would say that the seat of the stricture varies considerably in different cases; that in the simplest form it is nothing more than a contracted state of the os uteri externum; but in other instances it appears to be formed by the inferior segment of the uterus itself," \&e.

I have written in italics those symptoms in my patient's 